\title{
Purity of Plasma as a Sample for Neuropsychiatric Omics Studies Based on Virchow's Cellular Principle
}

\section{Noriyuki Kawamura*}

Kawamura Clinic for General Practice, Gyouki-Kai Medical Corporation, Tokyo, Japan

\begin{abstract}
As an idea to ensure the success of OMICS research, there is Virchow's pathological ideas of physical disease. This idea is that one cell, a group of cells, a substance, and a group of substances are specific for each disease. Based on this idea, it can be inferred that the unity and purity of the sample and the disease used are extremely important factors. In this study, the aim is to show how the difference in trivial centrifugal conditions of whole blood affected the plasma concentration of Phosphoethanolamine (PEA), a biomarker of depression shown in our previous study, It turned out that the value of PEA increases when the rotor is a swing type, or when the temperature at the time of centrifugation is set at $4^{\circ} \mathrm{C}$. This increase might be due to platelet contamination in isolated plasma. Therefore, especially when the researchers are looking to discover an unknown marker, special attention should be paid to sample.
\end{abstract}

Keywords: Psychiatric disorders; Virchow's cellular principle; Plasma

\section{Introduction}

Thanks to Virchow's cellular principle [1], we were able to show that Major Depressive Disorder (MDD) with low plasma Phosphoethanolamine (PEA) concentration [2] is a central feature of MDD [3] and appears as a main group of MDD with psychomotor retardation and cognitive impairment [4].

If psychiatric disorders were physical diseases, it would have been reasonable to search for biomarkers by OMICS technologies. Moreover, it may be impossible to search for the biomarkers if psychiatric disorders were not diseases, but just behavioral abnormalities [5], it is likely that different coherent groups of substance would be captured. However, recent Treatment Guidelines of Depression [6] have been created on the premise that there is little doubt about the effectiveness of antidepressants in moderate or severe depression $[7,8]$. This means that several forms of depression can be considered as disease of the brain like various specific physical organ diseases. It would therefore be reasonably and beneficial to hypothesize a bulk of psychiatric disorders as physical diseases.

The two major premises of Virchow's cellular principle [1] are: I) A physical disease is characterized only by pathological findings that are absent in other diseases; and II) A specific group of cells or substances are associated with the pathology of that disease. This thought process has driven the extensive modern medical advances in the world. For instance, a new classification of viral hepatitis and four classifications for neurodegenerative dementias including Alzheimer's disease, Parkinson's disease, frontotemporal dementia and amyotrophic lateral sclerosis; are good examples that psychiatry must learn from. It cannot be assumed that MDD is only one disease, it is thought to be a complex of several diseases with different pathologies. One pathology common to these different pathologies would be interpreted as a decrease in PEA.

In order to apply Virchow's principles to psychiatric biomarker discovery, there are two inevitable tasks that must be satisfied simultaneously: For principle I), one must identify a pure depression group that is independent of other diseases and disorders. Also, to satisfy principle II), the purity of the biological sample handled for OMICS studies should be extremely high.

To fulfill principle I) for the search of MDD biomarkers, it is necessary to clear the elimination criteria of C. D. and E. for MDD in DSM-IV or -5 [9,10]. Besides, it is necessary to emphasize the distinction between General Anxiety Disorder (GAD) and MDD. A new specifier added to DSM-5 due to the presence or absence of anxious distress is a significant diagnostic progress [10].

To fulfill principle II), one needs to thoroughly eliminate substances derived from other cellular components. We therefore focused on purifying the samples used in the present study. If the sample is plasma, we must go back to the definition. According to encyclopedia Britanica, the definition of plasma is the liquid portion of blood and also written thus, "Plasma is derived when all the blood cells erythrocytes, leukocytes and platelets are separated from whole blood." [11]

The aim of the present study is to improve reproducibility of the previous study by other researchers. We evaluated plasma acquisition methods based on comparison of PEA concentrations. We hereby share a range of information obtained from this study.

\section{Methods}

\section{Human subjects}

The 14 subjects were recruited from the patients who visited the Kawamura Clinic for General Practice (KCGP). Patients were provided with written informed consent for participation in the study under the approval of the KCGP Institutional Ethics Committee.

\section{Blood sampling}

Peripheral blood samples were collected into one $2 \mathrm{~mL}$ and three 5 mL EDTA-2Na vacuum tubes (Terumo, Tokyo, Japan). Platelet count was measured by multi-item automatic blood cell analyzer XN-330 (Sysmex, Osaka, Japan). Plasma was separated out by centrifugation, at $870 \mathrm{~g}$ for $15 \mathrm{~min}$ with a swing-type rotor H19-a (Kokusan Co., Ltd., Tokyo, Japan) and that then with an angled-type rotor MX301 (Tommy Seiko Co., Ltd., Tokyo, Japan), respectively.

*Corresponding author: Noriyuki Kawamura, Kawamura Clinic for General Prac tice, Gyouki-Kai Medical Corporation, Tokyo, Japan, Tel: +81-3-3478-1146, E-mail: nkawamura@g-clinic.net

\section{Received April 17, 2018; Accepted July 31, 2018; Published August 05, 2018}

Citation: Kawamura N (2018) Purity of Plasma as a Sample for Neuropsychiatric Omics Studies Based on Virchow's Cellular Principle. J Neurol Neurophysiol 9 467. doi:10.4172/2155-9562.1000467

Copyright: ( $) 2018$ Kawamura N. This is an open-access article distributed unde the terms of the Creative Commons Attribution License, which permits unrestricted use, distribution, and reproduction in any medium, provided the original author and source are credited. 
To elucidate the effects of centrifugal conditions on the concentrations of plasma obtained, we set three conditions with the same centrifugational acceleration speed. For the control condition, denoted $\mathrm{AR}$, the whole blood samples were centrifuged at room temperature with a fixed angle-type rotor. The test conditions were denoted A4 and SR. For condition A4, the whole blood samples were centrifuged at $4^{\circ} \mathrm{C}$ with a fixed angle-type rotor. Condition SR included centrifugation of the whole blood samples at room temperature using swing-type rotor. One $\mathrm{mL}$ each of the upper layer portion and the lower layer portion of plasma was separated and collected into the storage tubes. All the samples were stored in liquid nitrogen immediately after processing.

\section{Targeted analysis of PEA using ion-chromatography}

Plasma PEA concentration was determined using an IonChromatography Fluorescence Detection (IC-FLD) method described previously [1] with some modifications. Briefly, plasma samples were deproteinated by centrifugation using a Nanosep centrifugal device with Omega membrane 3-kD cutoff filter (Pall Co., Port Washington, NY, USA) at $4^{\circ} \mathrm{C}$ and $9,100 \times g$ for approximately $2 \mathrm{~h}$. Prior to centrifugation, plasma samples $(50 \mu \mathrm{L})$ were diluted 10 -fold using MilliQ water (final volume $500 \mu \mathrm{L}$ ) including $1 \mu \mathrm{M}$ homocysteinic acid as an internal standard (IS). A portion of the filtrate $(10 \mu \mathrm{L})$ was applied to a Dionex ICS-5000 IC system (Thermo Fisher Scientific, Waltham, MA, USA) equipped with a thermoreactor Nanospace 3019 SI-2 (Shiseido, Tokyo, Japan), and a fluorescence detector UltiMate 3000 (Thermo Fisher Scientific). The system was operated by the Chromeleon 6.80 SR11 build 3161 software (184582; Thermo Fisher Scientific). PEA was separated using an anion-exchange column IonPac AS20 (Thermo Fisher Scientific) with an isocratic flow of $20 \mathrm{mM} \mathrm{NaOH}$ at $0.25 \mathrm{~mL} / \mathrm{min}$ and $35^{\circ} \mathrm{C}$. The post column eluents were mixed with a fluorescent solution including $0.5 \mathrm{mg} / \mathrm{mL} o$-phthalaldehyde and $0.1 \%$ $(v / v) 2$-mercaptoethanol at $0.1 \mathrm{~mL} / \mathrm{min}$ and incubated at $35^{\circ} \mathrm{C}$ through a reactor tube (i.d., $0.25 \mathrm{~mm}$; length, $5 \mathrm{~m}$ ). The fluorescent derivative of PEA and the IS, typically eluting at $7 \mathrm{~min}$ and $13 \mathrm{~min}$, respectively, was detected by the fluorescence detector with excitation and emission wavelengths of $340 \mathrm{~nm}$ and $450 \mathrm{~nm}$, respectively. The area value of PEA was normalized by the area value of the internal standard.

\section{Statistical analysis}

Data was analyzed with IBM SPSS version 23. The control condition $\mathrm{AR}$, was compared with other 2 test conditions by paired $t$ test. We did not conduct comparisons between A4 and SR because of many covariates. By PS program version 3.0 (provide freely by. D. Dupont and W. D. Plummer), it was clear that 14 subjects were enough for paired t-test.

Kendall tau $\mathrm{b}$ analyses between platelet count and PEA values were done to see if there are effects of the residual platelets in the plasma. Power can be seen by Kendall tau b value.

\section{Results}

\section{Subjects}

The subjects included 14 patients with major depression (6 persons) and without major depression (8 persons). The mean and standard error of all the subjects were 43.5 years old and 9.85 respectively. Those of 5 male subjects were 48.4 years old and 6.11 and those of 9 female subjects were 40.8 years old and 10.76 .

\section{The comparisons by paired $t$ test}

The raw data of the PEA concentration of plasma are shown in
Table 1. PEA concentrations of A4-1 was significantly higher than those of AR- 1 ( $\mathrm{t}$ value $=7.63, \mathrm{df}=13$ ). The significant differences were similarly observed between A4-2 and AR-2, A4-m and AR-m ( $t$ value $=8.13$ $\mathrm{df}=13, \mathrm{t}$ value $=7.92 \mathrm{df}=13$ respectively). PEA concentrations of SR-1, SR-2, SR-m were similarly observed higher than those of AR-1, AR-2 and $A R-m$ ( $t$ value $=9.25 \mathrm{df}=13, \mathrm{t}$ value $=9.94 \mathrm{df}=13$, and $\mathrm{t}$ value $=9.79$ $\mathrm{df}=13$ respectively). All $\mathrm{p}$ values of above six comparisons by paired $\mathrm{t}$ test were less than 0.001 . Thus, centrifugation at $4^{\circ} \mathrm{C}$ and centrifugation by swing-type rotor would be the factors that increased the values of plasma PEA.

The comparisons between the upper layer and the lower layer of the plasma showed that PEA concentrations of SR2 was significantly higher than $\mathrm{SR} 1$ by paired $\mathrm{t}$ test $(\mathrm{df}=13, \mathrm{P}=0.009, \mathrm{t}$ value 3.08 ). Among A4 series and AR series, there were no significant differences. This means there was a concentration gradient of PEA in the plasma separated by swing rotor centrifugation.

However, PEA concentrations of A4-2 was were higher but not significantly than $\mathrm{A} 4-1$ by paired $\mathrm{t}$ test $(\mathrm{df}=13, \mathrm{P}=0.09, \mathrm{t}$ value 1.80). Although the difference did not reach the significant level due to small number of the subjects, the same tendency of making concentration gradient of PEA in the supernatant or plasma portion after centrifugation.

\section{Correlation of platelet count and PEA concentrations}

Though both the platelet count and PEA concentrations are quantitative variables, due to limited number of the subjects, it was impossible to assume that they are normally distributed. Using Kendall's tau b correlation analysis, plasma PEA concentrations of AR, AR-1, AR-2, A4, A4-1, and A4-2 did not correlated significantly with platelet count. However, SR-1, SR-2 and SR-m correlated significantly with platelet count (Kendall's tau $b=0.54,0.63,0.63$, and all $p$ values were lower than 0.01 ). This indicated the strong possibility that platelets still remained in the plasma separated by swing rotor centrifugation.

Among the correlation analyses between the platelet count and the subtraction differences of (A4 series - AR series) and (SR series - AR series), platelet count correlated strongly and significantly with (A4-1 AR-1), (SR-1 - AR-1), (SR-2 - AR-2) and (SR-m - AR-m) (Kendall's tau $\mathrm{b}=0.41,0.50,0.58$ and 0.60 ). These results indicated the possibility that platelet remaining in the upper portion of plasma separated at $4^{\circ} \mathrm{C}$ have increased PEA concentrations than at room temperature even with the fixed angle-type rotor. Additionally, effects of the remaining platelets might have happened at any portion of the plasma due to swing-type centrifugation.

\begin{tabular}{|c|c|c|c|}
\hline \multirow{2}{*}{\multicolumn{2}{|c|}{$\begin{array}{l}\text { Centrifuge condition and the upper }(-1) \text { and the } \\
\text { lower }(-2) \text { layer of plasma }\end{array}$}} & \multicolumn{2}{|c|}{ PHA concentration $(\mu \mathrm{M})$} \\
\hline & & \multirow{2}{*}{$\begin{array}{c}\text { Mean } \\
1.65\end{array}$} & \multirow{2}{*}{$\begin{array}{l}\text { SD } \\
0.54\end{array}$} \\
\hline \multirow{3}{*}{$\begin{array}{l}\text { Using Angled-type rotor and at } \\
\text { Room temperature (AR) }\end{array}$} & AR-1* & & \\
\hline & AR-2* & 1.66 & 0.54 \\
\hline & AR-m** & 1.65 & 0.53 \\
\hline \multirow{3}{*}{$\begin{array}{l}\text { Using Angled-type rotor and at } 4^{\circ} \mathrm{C} \\
\text { (A4) }\end{array}$} & $\mathrm{A} 4-1^{*}$ & 2.29 & 0.71 \\
\hline & $\mathrm{A} 4-2^{*}$ & 2.33 & 0.68 \\
\hline & $\mathrm{A} 4-\mathrm{m}^{* *}$ & 2.31 & 0.68 \\
\hline \multirow{3}{*}{$\begin{array}{l}\text { Using Swing-type rotor and at } \\
\text { Room temperature (SR) }\end{array}$} & SR-1* & 3.30 & 0.87 \\
\hline & SR-2* & 3.52 & 0.97 \\
\hline & SR-m** & 3.41 & 0.91 \\
\hline
\end{tabular}

Table 1: Mean and SD of plasma PEA values for each condition $\left({ }^{*}\right.$ Each of the upper layer portion and lower layer portion was named as -1 and -2 . ${ }^{* \star}$ The mean values of PEA concentration of the upper portion and lower portion of each plasma was named as AR-m, A4-m and SR-m, respectively). 
Citation: Kawamura N (2018) Purity of Plasma as a Sample for Neuropsychiatric Omics Studies Based on Virchow's Cellular Principle. J Neurol Neurophysiol 9: 467. doi:10.4172/2155-9562.1000467

\section{Discussion}

\section{Possible adverse effects of platelets remaining in plasma on for OMICS research}

PEA is a very small molecule and should be homogeneously distributed in aqueous solution such as plasma. It is unlikely that a gradient will be born occur in the plasma under weak centrifugation conditions that segregate the plasma. If a gradient of PEA is born exists, PEA can only be considered if it is in some kind of granules or cells. Structures capable of forming PEA gradient of PEA are of course considered lymphocytes and others, but we assume that platelets are most dubious as discussed in this study. Dense granules are present in platelets and include Adenosine Di-Phosphate (ADP), ATP, serotonin, calcium ion, and so on [12]. These small molecules are absorbed into the granules from the blood. Also, a granules are present, and include fibrinogen, von Willebrand Factor (vWF), coagulation factor V, and other proteins [12]. Obviously, platelet contamination may likely interfere with plasma OMICS studies. Although we did not measure the numbers of platelet remaining in plasma, low centrifugation temperature such as $4^{\circ} \mathrm{C}$ during centrifugation and centrifugation with swing-type rotor might cause increased platelet contaminations of plasma. The results of this study suggest that researchers should take sample preparation and centrifugation parameters into consideration and be aware that platelets remaining in the plasma might alter the plasma concentrations of PEA and other molecules, so as to measure the PEA concentration and also for best results in plasma-based metabolomics as well as other OMICS technologies. We emphasize that plasma should be prepared by centrifugation at room temperature using fixed angle-type rotors because of our experiences. This condition has greatest possibility to minimize platelet contamination of plasma. It is therefore safe to say that underestimation of this basic centrifugation condition may underlie the reasons why other researchers have not found the importance of PEA in MDD by OMICIS technologies; hence/ and the differences of values of the plasma PEA in the literature [1].

\section{Future achievement directions by emphasizing the cellular principle}

By the same token, careful analysis and application of Virchow's cell theory to subject selection and sample preparation in OMICS including genomics, would likely yield fruitful results for the future. In the future, in order to move the body disease hypothesis of psychiatric disorders forward, neuropsychiatric diseases could be expressed by mathematical matrix display as described in the supporting document of our previous work [1]. Future attempts could employ profiling matrices organized according to metabolic pathways, genetic predisposition, and protein/ peptide signatures to make objective diagnostic and clinical decisions.

Such a psychiatric matrix could be combined with data on general physical health, medications, and life style factors, presently there is no such formula in clinical utility due to limited information; we are however hopeful without giving up, a time will come when psychiatric diseases will be diagnosed and managed like other physical diseases.

\section{Acknowledgements}

My sincere appreciation to the staff of Human Metabolome Technologies Inc, Ms. Aya Hoshi and Ms. Kaori Hanawa for their assistance with IC-FLD measurement of plasma phosphoethanolamine; Thanks to Dr. Yoshiaki Ohashi for critical advise on targeted metabolomics; and to Dr. Douglas Osei-Hyiaman for conceptual advise, proofreading and editing of the manuscript.

\section{References}

1. Virchow R (1858) Cellular pathology in its justification on physiological and pathological tissue theory.

2. Kawamura N, Shinoda K, Sato H, Sasaki K, Suzuki M, et al. (2018) Plasma metabolome analysis of patients with major depressive disorder. Psychiatry Clin Neurosci 72: 349-361.

3. van Loo HM, de Jonge P, Romeijn JW, Kessler RC, Schoevers RA (2012) Datadriven subtypes of major depressive disorder: A systematic review. BMC Med 10: 156.

4. Bennabi D, Vandel P, Papaxanthis C, Pozzo T, Haffen E (2013) Psychomotor retardation in depression: A systematic review of diagnostic, pathophysiologic and therapeutic implications. BioMed Res Int 2013: 158746.

5. Szasz T (2008) Psychiatry: The science of lies. Syracuse university press, pp: $2-5$

6. The preparation committee for mood disorder treatment guideline (2017) Japan depression society treatment guidelines of depression (DSM-5)/major depressive disorder. 2nd Edition.

7. Arroll B, Elley CR, Fishman T, Goodyear-Smith FA, Kenealy T, et al. (2009) Antidepressants versus placebo for depression in primary care. Cochrane Database Syst Rev, p: CD007954.

8. Baghai TC, Blier P, Baldwin DS (2012) Executive summary of the report by the WPA section on pharmacopsychiatry on general and comparative efficacy and effectiveness of antidepressants in the acute treatment of depressive disorders. Eur Arch Psychiatry Clin Neurosci 262: 13-22.

9. American psychiatric association (2000) Diagnostic and statistical manual of mental disorders: DSM-IV TR.

10. American psychiatric association (2013) Diagnostic and statistical manual of mental disorders: DSM-5.

11. Yawn D (2017) Plasma biology.

12. Sharda A, Flaumenhaft R (2018) The life cycle of platelet granules. F1000Research. 